\title{
A Highly Stable, Low-Cost Metal-Free Oxygen Reduction Reaction Electrocatalyst Based on Nitrogen-Doped Pseudo-Graphite.
}

Kailash Hamal$^{1}$, Jeremy May ${ }^{1}$, Dipak Koirala ${ }^{1}$, Haoyu Zhu ${ }^{1}$, Humayun Kabir ${ }^{1}$, Elena Echeverria $^{2}$, David N. McIlroy ${ }^{2}$, Nolan Nicholas, ${ }^{3}$ I. Francis Cheng ${ }^{1 *}$

${ }^{1}$ Department of Chemistry, University of Idaho, 875 Perimeter Dr, MS 2343, Moscow, ID 83844, USA

${ }^{2}$ Department of Physics, Oklahoma State University, 145 Physical Sciences Building, Stillwater, OK 74028, USA

${ }^{3}$ ABB Future Labs, 940 Main Campus Drive, STE 200, Raleigh, NC 27606

*Corresponding author: Tel: (208) 885-6387, E-mail: ifcheng@uidaho.edu (I. Francis Cheng) 
Particle loading study: A particle loading of $1.0 \mathrm{mg} \mathrm{cm}^{-2}$ of N'-GUITAR/KB was used in this study. This loading had the highest ORR current density at $-0.2 \mathrm{~V}$ vs $\mathrm{Ag} / \mathrm{AgCl}$ relative to others shown in Figure $\mathrm{S} 1$. In general, $\mathrm{j}_{\mathrm{p}}$ increases with higher particle loadings, however, there is a negative shift in $E_{p}$ with higher particle loadings. This is possibly from increased contact resistance between the particles at higher loadings.

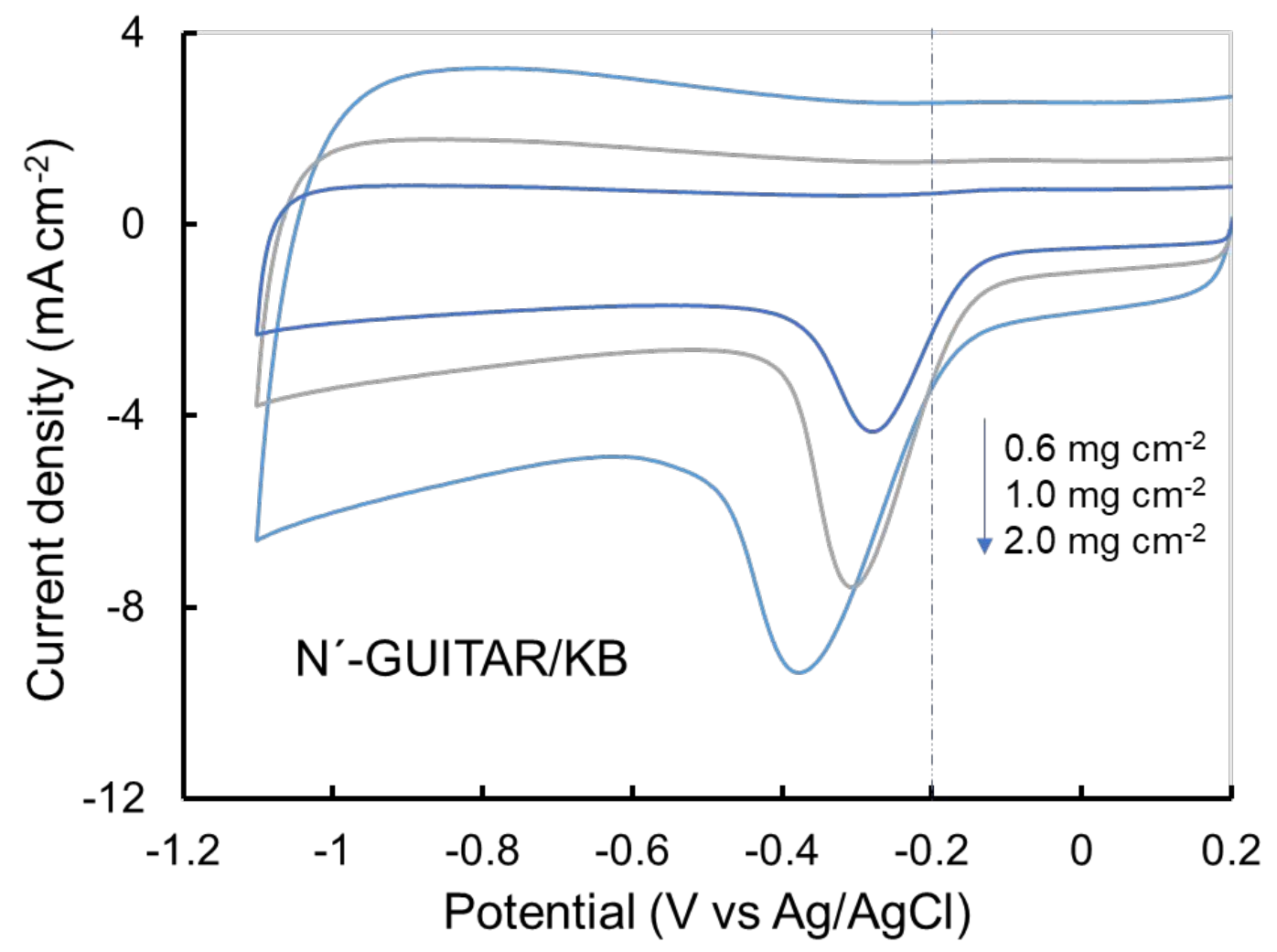

Figure S1: Particle loading study on $\mathrm{N}^{\prime}$-GUITAR/KB. Electrodes were cycled in $\mathrm{O}_{2}$ saturated $0.1 \mathrm{M} \mathrm{KOH}$ at $0.05 \mathrm{~V} \mathrm{~s}^{-1}$. The mass \% of Nafion and volume of solvents were kept consistent during this experiment. 
XPS study on Ketjen black (KB) substrate and Nitrogen doped Ketjen black (N'-KB): XPS results of Ketjen black substrate and nitrogen doped Ketjen black are shown in Figure S2. N'-KB contains 0.6 atomic $\%$ nitrogen (Figure S2D). The deconvoluted N1s peak of $\mathrm{N}^{\prime}-\mathrm{KB}$ contains $17.2 \%$ of graphitic nitrogen (Figure S2F), whereas $\mathrm{N}^{\prime}$-GUITAR/KB contains none.

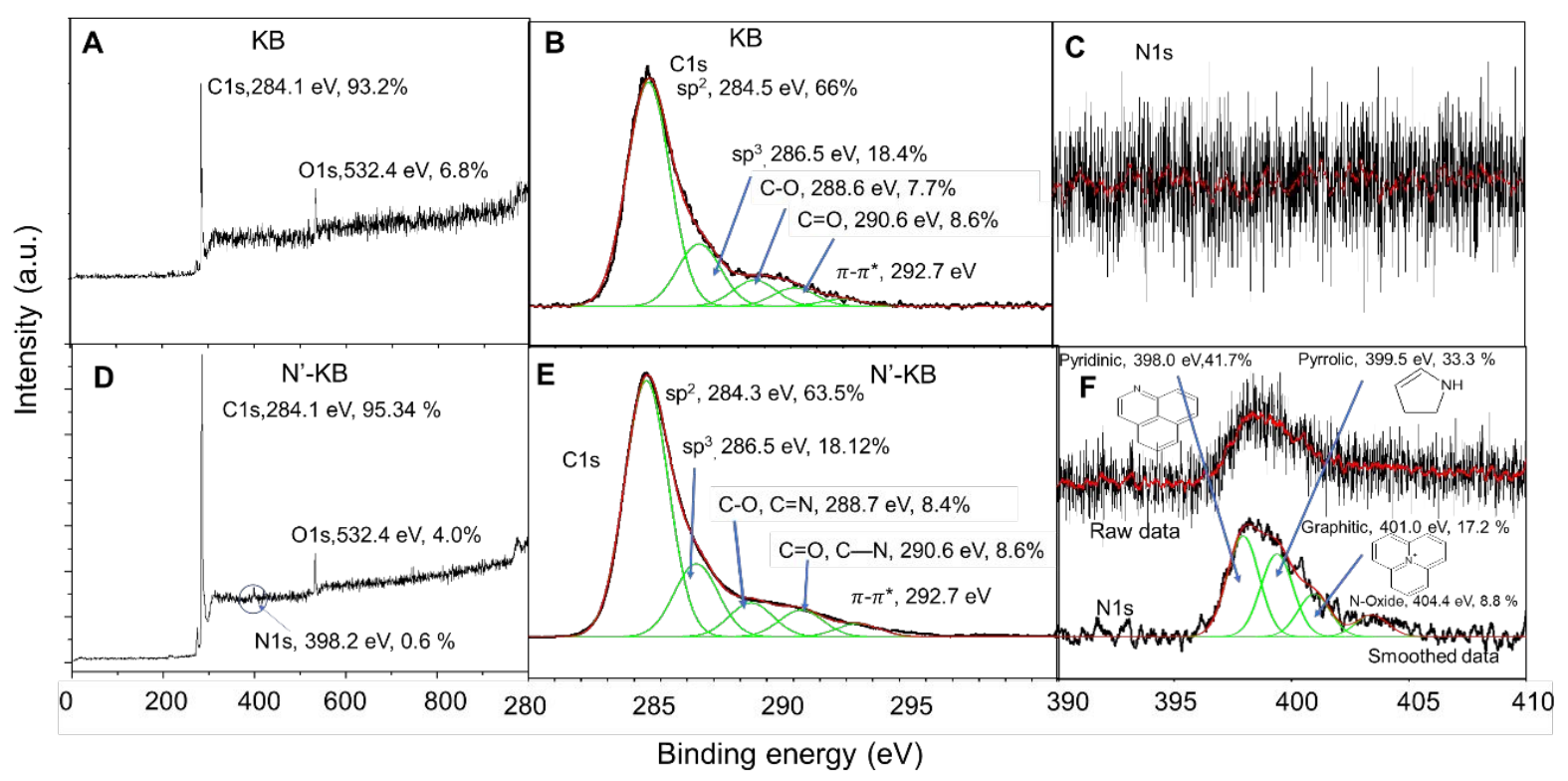

Figure S2: XPS comparison between KB and N'-KB. (A and D) Wide-scan XPS with the C1s, N1s, and O1s peaks highlighted. (Band E) High-resolution XPS of the C1s peak with deconvolved functional group peaks. (C and F) High-resolution XPS of the N1s peak with the deconvoluted peaks. 
The electrochemically active surface area (ECSA): ECSA for $\mathrm{N}^{\prime}$-GUITAR/KB is calculated from the non-faradaic capacitive current at $+0.1 \mathrm{~V}$ vs $\mathrm{Ag} / \mathrm{AgCl} .{ }^{1}$ This current was plotted vs the scan rates from 0.005 to $0.3 \mathrm{~V} \mathrm{~s}^{-1}$ (Figure S2). The slope of this plot is the electrical double layer capacitance, which is $50.5 \mu \mathrm{F} \mathrm{cm}{ }^{-2}$ for flat GUITAR and $4460 \mu \mathrm{F} \mathrm{cm}^{-2}$ for $\mathrm{N}^{\prime}$-GUITAR/KB. The ECSA is calculated from Equation 1, where $\mathrm{C}_{\mathrm{dl}}$ is the capacitance for $\mathrm{N}^{\prime}$-GUITAR/KB, and $\mathrm{C}_{\mathrm{dl}}$, flat is for the flat GUITAR electrode.

$$
\left(E C S A=\frac{C_{d l}}{C_{d l, f l a t}}\right)
$$

The ECSA for $\mathrm{N}^{\prime}$-GUITAR/KB is $883.16 \mathrm{~cm}^{2}$ per $\mathrm{cm}^{2}$ of geometric area. It is assumed that 1 $\mathrm{cm}^{2}$ of flat GUITAR surface area is equal to $1 \mathrm{~cm}^{2}$ of ECSA, assuming a surface roughness factor of 1 .
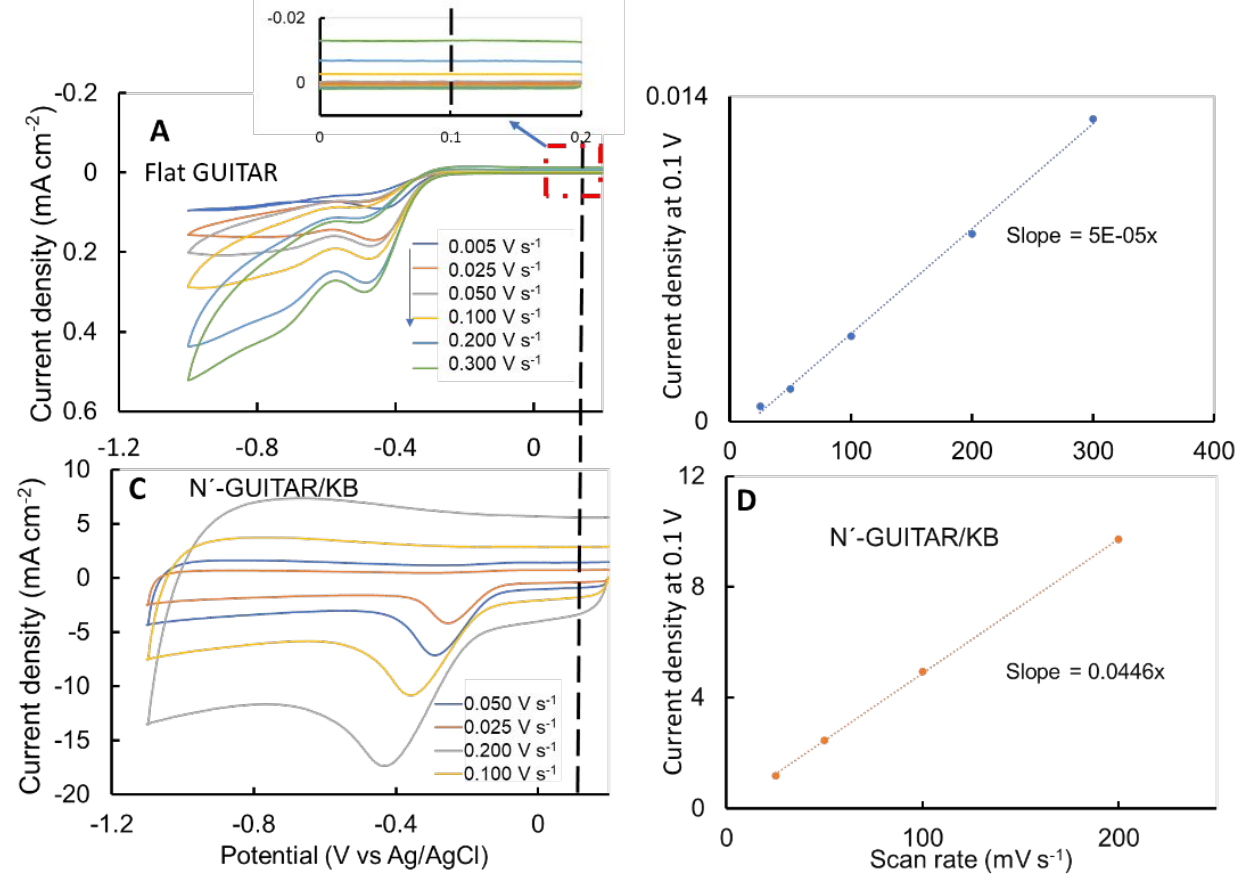

Figure S3: (A) and (C) include cyclic voltammograms at different scan rates on Flat GUITAR and $\mathrm{N}^{\prime}$-GUITAR/KB electrodes, respectively, in $\mathrm{O}_{2}$ purged $0.1 \mathrm{M} \mathrm{KOH}$. (B) and (D) are plots of non-faradic current density at $+0.1 \mathrm{~V}$ vs scan rate. The slope of these plots gives capacitive current. 
The electrochemically active surface area (ECSA): ECSA for Pt/C electrocatalyst is calculated from the hydrogen desorption method using equation 2 .

$$
\left(E C S A=\frac{\boldsymbol{Q} \text { total }\left(\mu C \mathrm{~cm}^{-2}\right)}{\boldsymbol{Q m o n o l a y e r}\left(\mu C \mathrm{~cm}^{-2}\right) * \text { mass }\left(\mathrm{g} \mathrm{m}^{-2}\right)}\right)
$$

Where $\mathrm{Q}_{\text {monolayer }}$ is $210 \mu \mathrm{C} \mathrm{cm}^{-2}$ and $\mathrm{Q}_{\text {total }}$ is the total charge for hydrogen desorption. ${ }^{2-5}$ The ECSA for $\mathrm{Pt} / \mathrm{C}$ is $57.9 \pm 0.3 \mathrm{~m}^{2} \mathrm{~g}^{-1}(\mathrm{n}=2)$. This area is shown in Figure $\mathrm{S} 4$.

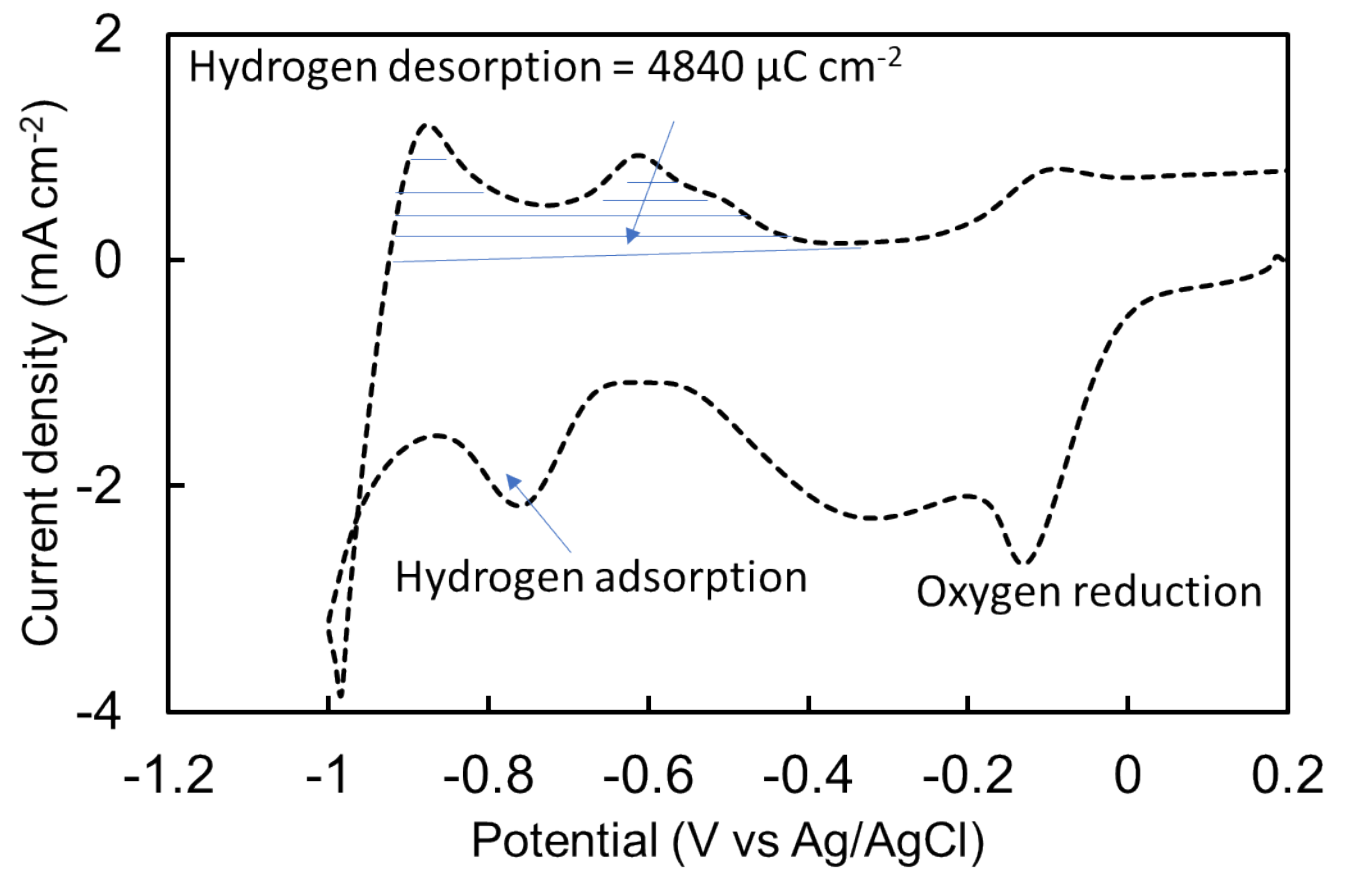

Figure S4: $\mathrm{Pt} / \mathrm{C} \mathrm{CV}$ in $0.1 \mathrm{M} \mathrm{KOH}$ at $0.05 \mathrm{~V} \mathrm{~s}^{-1}$. 
ORR performance of the control surfaces: The controls in these studies include unmodified Ketjen black and N-doped Ketjen black. These were evaluated for ORR performance under the same conditions as the $\mathrm{N}^{\prime}$-GUITAR/KB electrocatalyst. Figure S5 shows the CVs of these studies. These are included in Table 1.

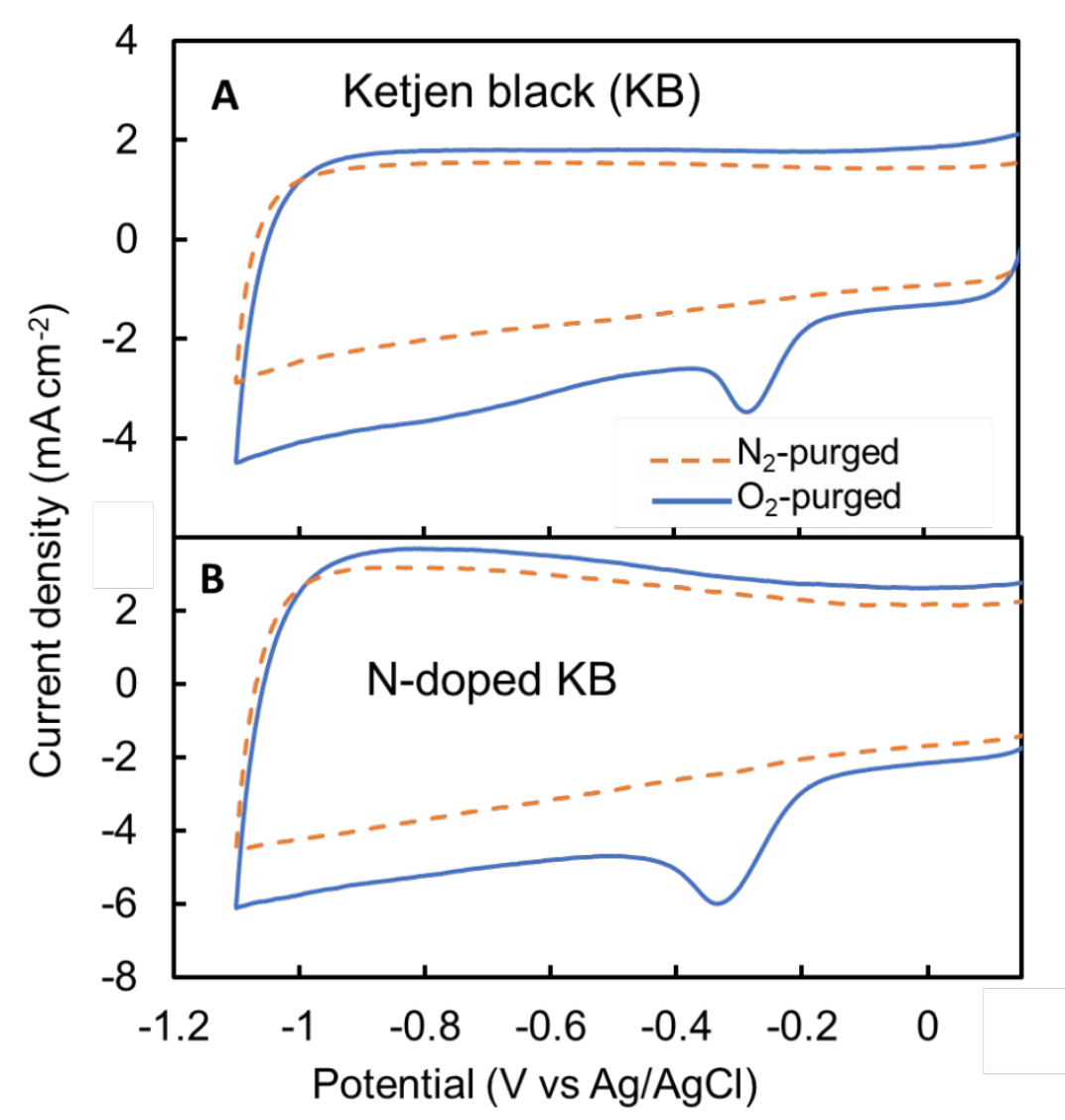

Figure S5: ORR CV at $0.05 \mathrm{~V} \mathrm{~s}^{-1}$ in $\mathrm{O}_{2}$ and $\mathrm{N}_{2}$ purged $0.1 \mathrm{M} \mathrm{KOH}$. A) Ketjen black and B) nitrogen doped Ketjen black ( $\left.\mathrm{N}^{\prime}-\mathrm{KB}\right)$. All electrodes consisted of Nafion composites with 1.0 $\mathrm{mg} \mathrm{cm} \mathrm{c}^{-2}$ particle loadings. 
ORR performance comparison with literature. The data for Figure 3B in the manuscript are shown in the following table with references.

Table S1: Literature showing ORR performance at $0.05 \mathrm{~V} \mathrm{~s}^{-1}$ in oxygen saturated $0.1 \mathrm{M} \mathrm{KOH}$. Note that $\mathrm{N}^{\prime}$-GUITAR/KB has a higher ORR current density at the peak potentials of the other electrocatalysts. Peak the current density $\left(\mathrm{j}_{\mathrm{p}}\right)$ based on electrode geometric surface area. (Note: *indicates values estimated from their data and description.)

\begin{tabular}{|c|c|c|c|}
\hline $\begin{array}{l}\text { Reference number in manuscript- (electrocatalyst- } \\
\text { journal- published year) }\end{array}$ & $\begin{array}{l}\text { Reference } \\
\text { in SI }\end{array}$ & $\begin{array}{l}\text { Peak } \\
\text { current } \\
\text { density }(\mathrm{jp}) \\
\left.(\mathrm{mA} \mathrm{cm})^{-2}\right)\end{array}$ & $\begin{array}{l}\text { Peak } \\
\text { potential } \\
\left(\mathrm{E}_{\mathrm{p}}\right)(\mathrm{V}) \mathrm{vs} \\
\mathrm{Ag} / \mathrm{AgCl}\end{array}$ \\
\hline $\mathrm{N}^{\prime}$-GUITAR/KB & TW & 6.27 & -0.270 \\
\hline $\begin{array}{l}\text { 16- (NCNP-CNF, ACS Appl. Mater. Interfaces- } \\
\text { 2016) }\end{array}$ & 6 & 3.20 & -0.280 \\
\hline $\begin{array}{l}\text { 23-(Fe,S/NGC-900, ACS Appl. Mater. Interfaces- } \\
\text { 2016) }\end{array}$ & 7 & $2.60^{*}$ & $-0.175^{*}$ \\
\hline $\begin{array}{l}\text { 30-(Co-N-OMMC 0.6, Applied Catalysis B: } \\
\text { Environmental-2016) }\end{array}$ & 8 & 2.51 & -0.195 \\
\hline $\begin{array}{l}\text { 24- (N, F-Carbon-1000, ACS Appl. Mater. } \\
\text { Interfaces- 2017) }\end{array}$ & 9 & $1.20^{*}$ & -0.147 \\
\hline 38-(Me/HNMK-5, ChemElectroChem-2018) & 10 & $1.20^{*}$ & $-0.225^{*}$ \\
\hline $\begin{array}{l}\text { 54-(Co@NS/CNT-MCF-900, J. of Materials } \\
\text { Chemistry A-2017) }\end{array}$ & 11 & $1.00^{*}$ & -0.146 \\
\hline 18-(NPCS-850-J. Phys. Chem. C-2018) & 12 & $1.50^{*}$ & -0.187 \\
\hline 56- (HT-NCTs, J. Mater. Chem. A- 2017) & 13 & $1.25^{*}$ & -0.266 \\
\hline 57-(PCPG-900, colloids and surfaceA-2018) & 14 & $1.20^{*}$ & $-0.275^{*}$ \\
\hline $\begin{array}{l}\text { 47-(CuAg@Ag/N-GNS-ACS Appl. Mater. } \\
\text { Interfaces-2018) }\end{array}$ & 15 & $2.20^{*}$ & -0.257 \\
\hline 58-(BP2000-NF, Scientific Reports-2013) & 16 & $2.60^{*}$ & -0.160 \\
\hline 45-(Co@N-CNR, ChemElectrochem-2018) & 17 & $1.20^{*}$ & -0.207 \\
\hline 55-( $\mathrm{Co}_{\mathrm{x}} \mathrm{O}_{\mathrm{y}} / \mathrm{CNT} @ \mathrm{C}$, Nano Research, 2016) & 18 & $3.00^{*}$ & -0.207 \\
\hline $\begin{array}{l}\text { 59-(3D-N-RGO/MnO, The Journal of Physical } \\
\text { Chemistry C-2015) }\end{array}$ & 19 & $1.00^{*}$ & -0.350 \\
\hline
\end{tabular}


Table S2: RDE-LSV comparison with literature. Comparison of half-wave potential $\left(\mathrm{E}_{1 / 2}\right)$ and limiting current densities $\left(\mathrm{j}_{\lim }\right)$ are shown in Figure 4B in the manuscript (Note: *indicates values estimated from their data and description). LSV conditions are $1600 \mathrm{rpm}$ at $0.005 \mathrm{~V} \mathrm{~s}^{-1}$ in $\mathrm{O}_{2}$ saturated $0.1 \mathrm{M} \mathrm{KOH}$ unless and otherwise mentioned.

\begin{tabular}{|c|c|c|c|c|}
\hline $\begin{array}{l}\text { Reference as in the } \\
\text { manuscript. } \\
\text { (electrocatalyst- } \\
\text { journal- published } \\
\text { year) }\end{array}$ & $\begin{array}{l}\text { Reference } \\
\text { in SI }\end{array}$ & $\begin{array}{l}\mathrm{E}_{1 / 2} \mathrm{~V} \\
\text { vs RHE }\end{array}$ & $\begin{array}{l}\text { Limiting } \\
\text { current } \\
\text { density, } \\
\left.(\mathrm{mA} \mathrm{cm})^{-2}\right)\end{array}$ & Stability \\
\hline N'-GUITAR/KB & TW & 0.82 & 6.3 & No loss till $30 \mathrm{~K}$ cycle \\
\hline $\mathrm{Pt} / \mathrm{C}$ & TW & 0.87 & 5.26 & $\begin{array}{l}-0.038 \mathrm{~V} \text { shift in } \mathrm{E}_{1 / 2} \text { after } \\
30000 \text { cycle } \\
-9 \% \text { current loss after } 30000 \\
\text { cycle } \\
-38 \% \text { loss in ECSA after } 30000 \\
\text { cycle }\end{array}$ \\
\hline \multicolumn{5}{|c|}{ Carbon based electrocatalysts } \\
\hline $\begin{array}{l}\text { 60. }\left(\mathrm{N}_{0.54}-\mathrm{Z}_{3} / \mathrm{M}_{1}^{-}\right. \\
\text {900, En.Env.Sci- } \\
\text { 2019) }\end{array}$ & 20 & 0.82 & 4.3 & $\begin{array}{l}-0.25 \mathrm{~mA} \mathrm{~cm} \mathrm{~cm}^{-2} \text { loss after } 1000 \\
\mathrm{CV} \text { test } \\
-2.6 \% \text { current decay over } 24 \mathrm{hr} \\
\text { at } 0.565 \mathrm{~V} \text { vs RHE }\end{array}$ \\
\hline $\begin{array}{l}\text { 61. (N-GNr@CNT, } \\
\text { Nature } \\
\text { communication- } \\
\text { 2018) }\end{array}$ & 21 & 0.839 & 5.1 & N/A \\
\hline $\begin{array}{l}\text { 62. (Porous carbon, } \\
\text { Energy Environ. } \\
\text { Sci.- 2014,) }\end{array}$ & 22 & 0.70 & 4.6 & $\begin{array}{l}-25 \% \text { current decay over } 6.9 \mathrm{hr} \\
\text { at } 0.4 \mathrm{~V} \text { vs RHE }\end{array}$ \\
\hline $\begin{array}{l}\text { 63. (N-Porous } \\
\text { carbon, } \\
\text { Nanomaterials - } \\
\text { 2020) }\end{array}$ & 23 & 0.76 & $3.6^{*}$ & $\begin{array}{l}-13.5 \% \text { current decay over } 10 \mathrm{hr} \\
\text { at } 0.8 \mathrm{~V} \text { vs RHE }\end{array}$ \\
\hline $\begin{array}{l}\text { 64. }\left(\mathrm{C}_{60}-\mathrm{SWCNT}, J .\right. \\
\text { Am. Chem. Soc.- } \\
\text { 2019) }\end{array}$ & 24 & 0.84 & 1.73 & $\begin{array}{l}-7.5 \% \text { current decay over } 50 \mathrm{hr} \\
\text { at } 0.7 \mathrm{~V} \text { vs RHE }\end{array}$ \\
\hline $\begin{array}{l}\text { 64. (NPDC-1.09, } \\
\text { Sustainable Energy } \\
\& \text { Fuels- 2019) }\end{array}$ & 25 & 0.84 & 6.01 & $\begin{array}{l}-11 \% \text { current decay over } 22 \mathrm{hr} \text { at } \\
0.8 \mathrm{~V} \text { vs RHE } \\
-0.015 \mathrm{~V} \text { shift after } 1000 \mathrm{CV} \text { test }\end{array}$ \\
\hline $\begin{array}{l}\text { 14.(N-GRW, } \\
\text { Science Advances - }\end{array}$ & 26 & 0.84 & 3.6 & $\begin{array}{l}-10 \% \text { current decay over } 12 \mathrm{hr} \text { at } \\
0.7 \mathrm{~V} \text { vs RHE }\end{array}$ \\
\hline
\end{tabular}




\begin{tabular}{|c|c|c|c|c|}
\hline 2016) & & & & $\begin{array}{l}-0.015 \mathrm{~V} \text { shift in } \mathrm{E}_{1 / 2} \text { after } 2000 \\
\text { cycle }\left(0.035 \mathrm{~V} \mathrm{E}_{1 / 2} \text { shift for }\right. \\
\mathrm{Pt} / \mathrm{C})\end{array}$ \\
\hline $\begin{array}{l}\text { 24. (N, F-Carbon- } \\
\text { 1000, ACS Appl. } \\
\text { Mater. Interfaces- } \\
\text { 2017) }\end{array}$ & 9 & 0.84 & 5.2 & $\begin{array}{l}-9 \% \text { current decay over } 5 \mathrm{hr} \text { use } \\
\text { at } 0.4 \mathrm{~V} \text { vs RHE } \\
-\mathrm{E}_{1 / 2} \text { shift by } 0.010 \mathrm{~V} \text { after } \\
10000 \mathrm{CV} \text { cycle }\end{array}$ \\
\hline $\begin{array}{l}\text { 56. (Hierarchically } \\
\text { tubular-NCTs, } \\
\text { journal of materials } \\
\text { chemistry A-2017) }\end{array}$ & 13 & 0.76 & 4.9 & $\begin{array}{l}-20 \% \text { current decay over } 50 \mathrm{hr} \\
\text { test at } 0.65 \mathrm{~V} \text { vs RHE }\end{array}$ \\
\hline $\begin{array}{l}\text { 66.(NGRO, ACS } \\
\text { Appl. Nano Mater. - } \\
\text { 2019,) }\end{array}$ & 27 & 0.84 & 2.5 & $\begin{array}{l}-\mathrm{E}_{1 / 2} \text { shift by } 0.020 \mathrm{~V} \text { shift after } \\
10000 \text { cycles }\end{array}$ \\
\hline $\begin{array}{l}\text { 78. (N-carbon } \\
\text { nanofibers N-CN, } \\
\text { nano energy-2015) }\end{array}$ & 28 & 0.8 & $5.6^{*}$ & $\begin{array}{l}-\mathrm{E}_{1 / 2} \text { shift by } 0.020 \mathrm{~V} \text { shift after } \\
10000 \text { cycles }\left(0.040 \mathrm{~V} \mathrm{E}_{1 / 2} \text { shift }\right. \\
\text { for } \mathrm{Pt} / \mathrm{C})\end{array}$ \\
\hline $\begin{array}{l}\text { 69.(Carbon foam } \\
\text { Angewandte } \\
\text { chemie- } 2019)\end{array}$ & 29 & 0.87 & $4.2^{*}$ & $\begin{array}{l}-20 \% \text { current decay over } 8.3 \mathrm{hr} \\
\text { at } 0.87 \mathrm{~V} \text { vs RHE }\end{array}$ \\
\hline $\begin{array}{l}\text { 39.(N-GNR8.3, } \\
\text { ACS Appl. Mater. } \\
\text { Interfaces- 2014,) }\end{array}$ & 30 & N/A & $3.5^{*}$ & $\begin{array}{l}-4.9 \% \text { current decay over } 1.38 \mathrm{hr} \\
\text { at }-0.3 \mathrm{~V} \text { vs } \mathrm{Ag} / \mathrm{AgCl}\end{array}$ \\
\hline \multicolumn{5}{|c|}{ Metal based electrocatalysts } \\
\hline $\begin{array}{l}\text { 67. (Atomically } \\
\text { dispersed } \mathrm{Zn}-\mathrm{N}-\mathrm{C} \text {, } \\
\text { Angew. Chem. Int. } \\
\text { Ed.- 2019) }\end{array}$ & 31 & 0.87 & 5.0 & $\begin{array}{l}-\mathrm{E}_{1 / 2} \text { shift by } 0.018 \mathrm{~V} \text { shift after } \\
10000 \text { cycle }\end{array}$ \\
\hline $\begin{array}{l}\text { 68.(10Co-N@DCN } \\
\text { F Angew. Chem. In. } \\
\text { Ed.- 2020) }\end{array}$ & 32 & 0.83 & 6.36 & $\begin{array}{l}-\mathrm{E}_{1 / 2} \text { shift by } 0.010 \mathrm{~V} \text { after } 5000 \\
\text { cycle } \\
-5 \% \text { current decay over } 8.3 \mathrm{hr} \text { at } \\
0.6 \mathrm{~V} \text { vs RHE }\end{array}$ \\
\hline $\begin{array}{l}\text { 70. }(\mathrm{CAN}-\mathrm{Pc}(\mathrm{Fe} / \mathrm{Co}) \\
\text {, Angewandte- } \\
2019)\end{array}$ & 33 & 0.84 & 5.23 & $\begin{array}{l}-25.4 \% \text { current decay over } 3.3 \mathrm{hr} \\
\text { at } 0.85 \mathrm{~V} \text { vs RHE }\end{array}$ \\
\hline $\begin{array}{l}\text { 71.(Cu-based SACs, } \\
\text { J. Mater. Chem. } A- \\
\text { 2019) }\end{array}$ & 34 & 0.81 & $5.8^{*}$ & $\begin{array}{l}-3.5 \% \text { current decay over } 16 \\
\text { min. } \\
-E_{1 / 2} \text { shift by } 0.009 \mathrm{~V} \text { shift after } \\
5000 \text { cycles }\end{array}$ \\
\hline $\begin{array}{l}\text { 72.(mesoporous,3D- } \\
\text { Co-N-C, J. Mater. } \\
\text { Chem. A- 2018) }\end{array}$ & 35 & 0.83 & $5.9^{*}$ & $\begin{array}{l}-\mathrm{E}_{1 / 2} \text { shift by } 0.022 \mathrm{~V} \text { after } 5000 \\
\text { cycles }\end{array}$ \\
\hline $\begin{array}{l}\text { 54. (Co-doped with } \\
\mathrm{N} \text { and S, J. Mater. } \\
\text { Chem. A- 2017) }\end{array}$ & 11 & 0.837 & $5.35^{*}$ & $\begin{array}{l}-\mathrm{E}_{1 / 2} \text { shift by } 0.005 \mathrm{~V} \text { after } 2000 \\
\text { cycle test }\left(0.055 \mathrm{~V} \mathrm{E}_{1 / 2} \text { shift for }\right. \\
\mathrm{Pt} / \mathrm{C})\end{array}$ \\
\hline
\end{tabular}




\begin{tabular}{|c|c|c|c|c|}
\hline & & & & $\begin{array}{l}-8.1 \% \text { current decay over } 24 \mathrm{hr} \\
\text { at } 0.8 \mathrm{~V} \text { vs RHE }\end{array}$ \\
\hline $\begin{array}{l}\text { 73. (AgPdPt } \\
\text { Nanotubes, ACS } \\
\text { Appl. Nano Mater.- } \\
\text { 2019) }\end{array}$ & 36 & 0.90 & 5.2 & $\begin{array}{l}-\mathrm{E}_{1 / 2} \text { shift by } 0.011 \mathrm{~V} \text { after } 5000 \\
\text { cycle } \\
-21.5 \% \text { current decay over } 5 \mathrm{hr} \\
\text { at } 0.5 \mathrm{~V}(\mathrm{Pt} / \mathrm{C} 6 \% \text { decrease in } \\
\text { current and, }>0.011 \mathrm{~V} \text { shift in } \\
\left.\mathrm{E}_{1 / 2}\right)\end{array}$ \\
\hline $\begin{array}{l}\text { 77. }\left(\mathrm{Fe}_{3} \mathrm{C}-\right. \\
\text { Encapsulated } \mathrm{Fe}-\mathrm{N}- \\
\text { Doped Carbon, } \\
\text { Langmuir - 2018) }\end{array}$ & 37 & 0.805 & $5.6 @ 0.275$ & $\begin{array}{l}-\mathrm{E}_{1 / 2} \text { shift by } 0.005 \mathrm{~V} \text { shift after } \\
10000 \text { cycle } \\
-8.3 \% \text { current decay over } 5.5 \mathrm{hr} \\
\text { test (For Pt/C } 0.072 \mathrm{~V}, 30 \% \mathrm{i}_{\mathrm{lim}} \\
\text { decrease.) }\end{array}$ \\
\hline $\begin{array}{l}\text { 30. (Cobalt-N Co } \\
\text { doped carbon, } \\
\text { Applied catalysis B; } \\
\text { Environmental - } \\
\text { 2016) }\end{array}$ & 8 & 0.83 & N/A & $\begin{array}{l}-\mathrm{E}_{1 / 2} \text { shift by } 0.003 \mathrm{~V} \text { after } 5000 \\
\text { cycle }(0.024 \mathrm{~V} \text { for } \mathrm{Pt} / \mathrm{C})\end{array}$ \\
\hline $\begin{array}{l}\text { 45. (Co-N-doped } \\
\text { carbon, } \\
\text { Chemelectrochem- } \\
\text { 2018) }\end{array}$ & 17 & N/A & N/A & $\begin{array}{l}-\mathrm{E}_{1 / 2} \text { shift by } 0.018 \mathrm{~V} \text { after } 3000 \\
\text { cycle }(0.045 \mathrm{~V} \text { for } \mathrm{Pt} / \mathrm{C})\end{array}$ \\
\hline
\end{tabular}

Tafel slope Analysis: 
Figure S6 Shows the Tafel slopes in the region in the low potential region above $-0.150 \mathrm{~V}$ are 0.110 and $0.098 \mathrm{~V} \mathrm{dec}^{-1}$ for $\mathrm{Pt} / \mathrm{C}$ and $\mathrm{N}^{\prime}$-GUITAR/KB, respectively. These are shown below with GUITAR/KB $\left(0.138 \mathrm{~V} \mathrm{dec}^{-1}\right)$.

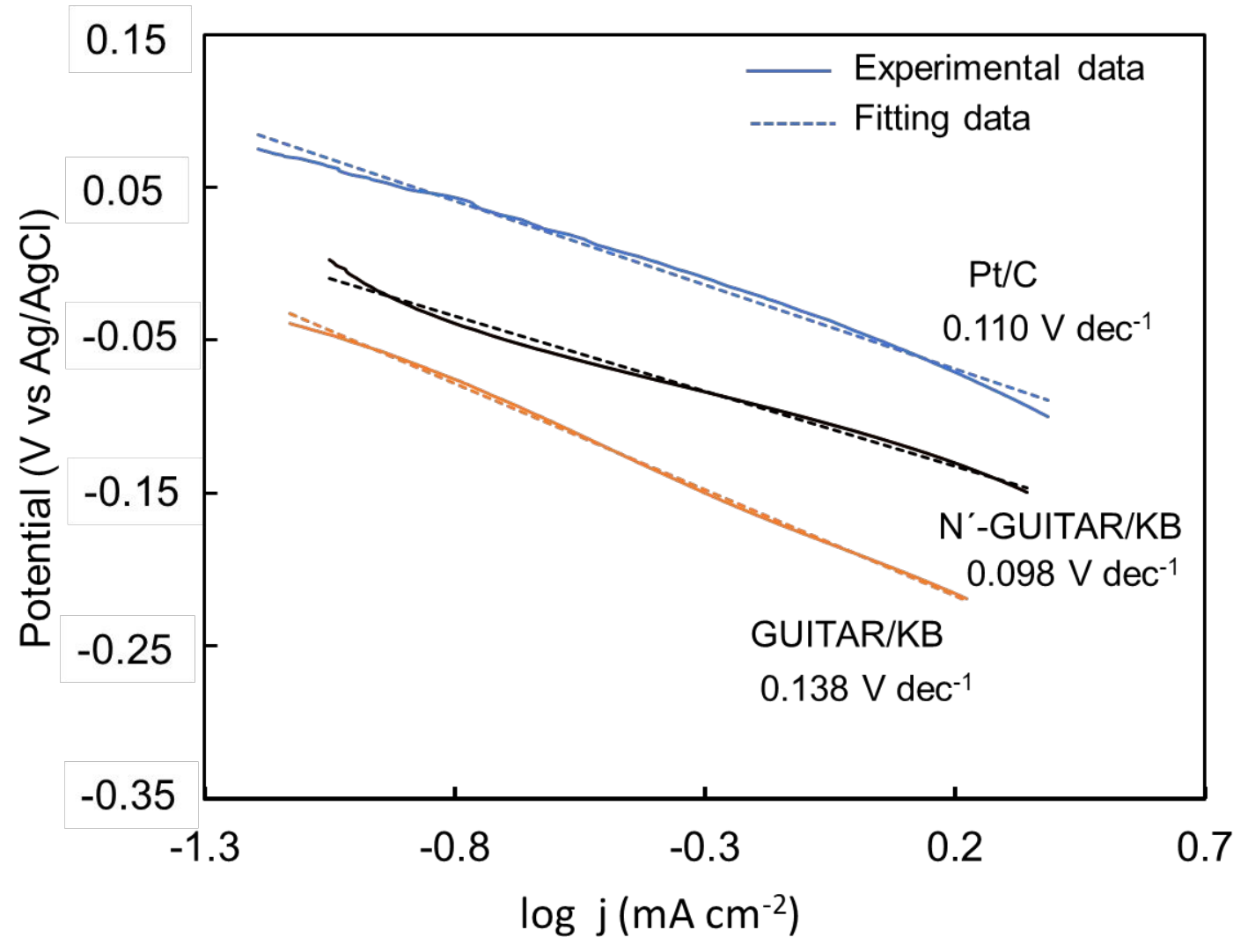

Figure S6: Tafel slopes for GUITAR/KB, N'-GUITAR/KB, and Pt/C. Slopes were calculated from ORR RDE LSV recorded at $1600 \mathrm{rpm}$ and $0.005 \mathrm{~V} \mathrm{~s}^{-1}$ as shown in Figure 4A before the stress test and Figure S7A.

Number of electron transfer (n) study for GUITAR/KB and Pt/C: 
Number of electron transfer during ORR was estimated from rotating disk electrode linear sweep voltammetry (RDE-LSV) at $0.005 \mathrm{~V} \mathrm{~s}^{-1}$ in $0.1 \mathrm{M} \mathrm{KOH}$ (Figures S7A and S8A). From the slope of Koutecky-Levich (K-L) plots, $\mathrm{n}=2.6$ to 2.8 for GUITAR/KB and $\mathrm{n}=3.6$ to 3.7 (Figure S7B, Equation 4 manuscript) for Pt/C within the potential range of $-0.4 \mathrm{~V}$ to $-0.7 \mathrm{~V}$ (Figure S8B).
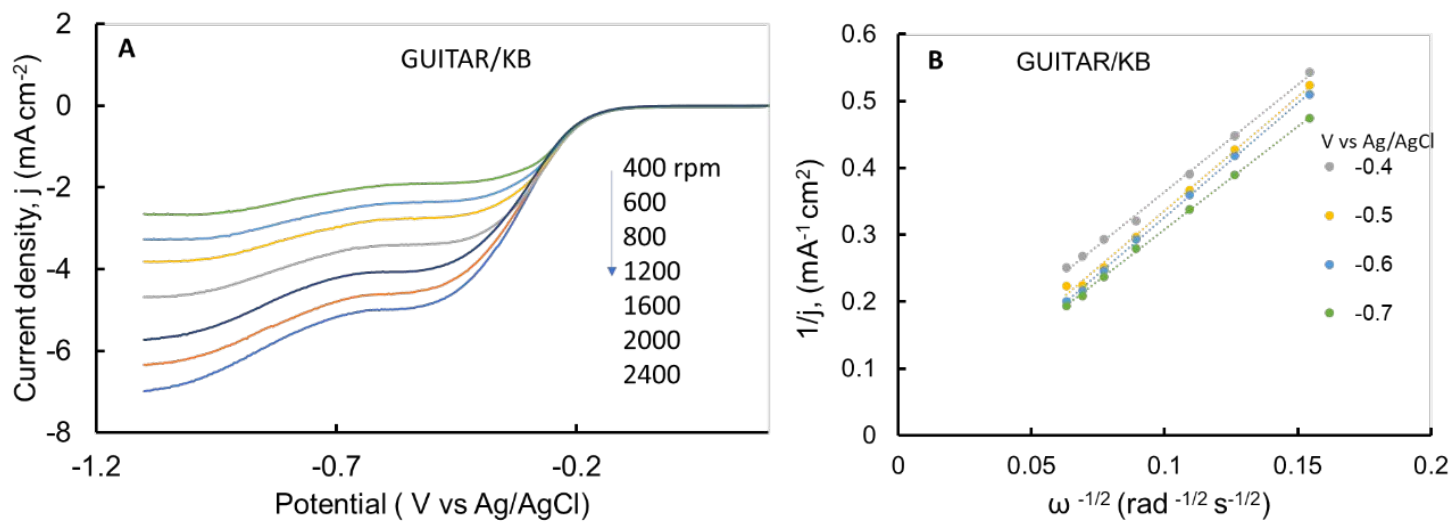

Figure S7: (A) Background corrected RDE-LSV in $\mathrm{O}_{2}$ saturated $0.1 \mathrm{M} \mathrm{KOH}$ at $0.005 \mathrm{~V} \mathrm{~s}^{-1}$ at different rotation speeds with GUITAR/KB. (B) K-L plot for GUITAR/KB.
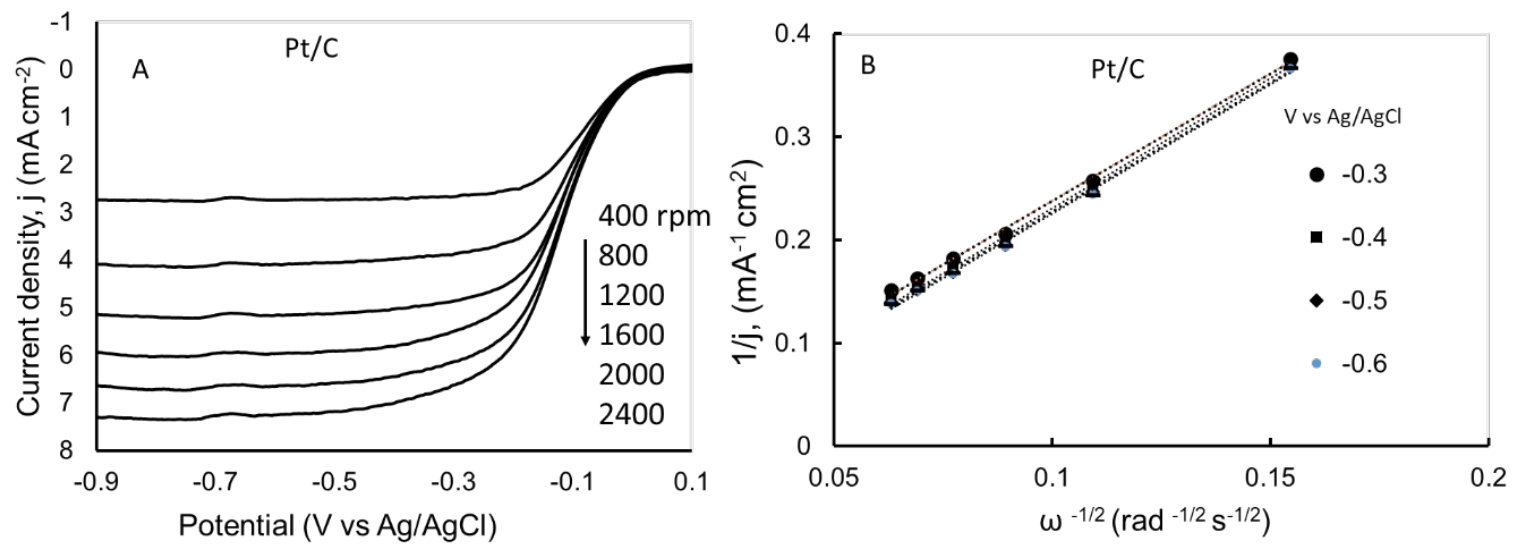

Figure S8: (A) Background corrected RDE-LSV in $\mathrm{O}_{2}$ saturated $0.1 \mathrm{M} \mathrm{KOH}$ at $0.005 \mathrm{~V} \mathrm{~s}^{-1}$ at different rotation speeds (rpm-rotations per minute) with Pt/C. (B) K-L plot for Pt/C.

\section{References:}


(1) Voiry, D.; Chhowalla, M.; Gogotsi, Y.; Kotov, N. A.; Li, Y.; Penner, R. M.; Schaak, R. E.; Weiss, P. S. Best Practices for Reporting Electrocatalytic Performance of Nanomaterials. ACS Nano 2018, 12 (10), 9635-9638. https://doi.org/10.1021/acsnano.8b07700.

(2) Garsany, Y.; Baturina, O. A.; Swider-Lyons, K. E.; Kocha, S. S. Experimental Methods for Quantifying the Activity of Platinum Electrocatalysts for the Oxygen Reduction Reaction. Anal. Chem. 2010, 82 (15), 6321-6328. https://doi.org/10.1021/ac100306c.

(3) Lukaszewski, M. Electrochemical Methods of Real Surface Area Determination of Noble Metal Electrodes - an Overview. 2016. https://doi.org/10.20964/2016.06.71.

(4) Begum, H.; Kim, Y.-B. Improvement of Catalytic Activity of Platinum Nanoparticles Decorated Carbon Graphene Composite on Oxygen Electroreduction for Fuel Cells. Processes 2019, 7 (9), 586. https://doi.org/10.3390/pr7090586.

(5) Lim, B.; Jiang, M.; Camargo, P. H. C.; Cho, E. C.; Tao, J.; Lu, X.; Zhu, Y.; Xia, Y. Pd-Pt Bimetallic Nanodendrites with High Activity for Oxygen Reduction. Science 2009, 324 (5932), 1302-1305. https://doi.org/10.1126/science.1170377.

(6) Panomsuwan, G.; Saito, N.; Ishizaki, T. Nitrogen-Doped Carbon Nanoparticle-Carbon Nanofiber Composite as an Efficient Metal-Free Cathode Catalyst for Oxygen Reduction Reaction. ACS Appl. Mater. Interfaces 2016, 8 (11), 6962-6971. https://doi.org/10.1021/acsami.5b10493.

(7) Men, B.; Sun, Y.; Liu, J.; Tang, Y.; Chen, Y.; Wan, P.; Pan, J. Synergistically Enhanced Electrocatalytic Activity of Sandwich-like N-Doped Graphene/Carbon Nanosheets Decorated by Fe and S for Oxygen Reduction Reaction. ACS Appl. Mater. Interfaces 2016, 8 (30), 19533-19541. https://doi.org/10.1021/acsami.6b06329.

(8) Sun, T.; Xu, L.; Li, S.; Chai, W.; Huang, Y.; Yan, Y.; Chen, J. Cobalt-Nitrogen-Doped Ordered Macro-/Mesoporous Carbon for Highly Efficient Oxygen Reduction Reaction. Applied Catalysis B: Environmental 2016, 193, 1-8. https://doi.org/10.1016/j.apcatb.2016.04.006.

(9) Lv, Y.; Yang, L.; Cao, D. Nitrogen and Fluorine-Codoped Porous Carbons as Efficient Metal-Free Electrocatalysts for Oxygen Reduction Reaction in Fuel Cells. ACS Appl. Mater. Interfaces 2017, 9 (38), 32859-32867. https://doi.org/10.1021/acsami.7b11371.

(10) Zhong, H.; Zhang, S.; Jiang, J.; Li, D.; Tang, P.; Alonso-Vante, N.; Feng, Y. Improved Electrocatalytic Performance of Tailored Metal-Free Nitrogen-Doped Ordered Mesoporous Carbons for the Oxygen Reduction Reaction. ChemElectroChem 2018, 5 (14), 1899-1904. https://doi.org/10.1002/celc.201700910.

(11) Wang, Z.; Peng, S.; Hu, Y.; Li, L.; Yan, T.; Yang, G.; Ji, D.; Srinivasan, M.; Pan, Z.; Ramakrishna, S. Cobalt Nanoparticles Encapsulated in Carbon Nanotube-Grafted Nitrogen and Sulfur Co-Doped Multichannel Carbon Fibers as Efficient Bifunctional Oxygen Electrocatalysts. J. Mater. Chem. A 2017, 5 (10), 4949-4961. https://doi.org/10.1039/C6TA10291C.

(12) Qin, L.; Yuan, Y.; Wei, W.; Lv, W.; Niu, S.; He, Y.-B.; Zhai, D.; Kang, F.; Kim, J.-K.; Yang, Q.-H.; Lu, J. Graphene-Directed Formation of a Nitrogen-Doped Porous Carbon Sheet with High Catalytic Performance for the Oxygen Reduction Reaction. J. Phys. Chem. C 2018, 122 (25), 13508-13514. https://doi.org/10.1021/acs.jpcc.7b12327.

(13) Wei, W.; Ge, H.; Huang, L.; Kuang, M.; Al-Enizi, A. M.; Zhang, L.; Zheng, G. Hierarchically Tubular Nitrogen-Doped Carbon Structures for the Oxygen Reduction 
Reaction. J. Mater. Chem. A 2017, 5 (26), 13634-13638.

https://doi.org/10.1039/C7TA02658G.

(14) Huang, X.; Yin, X.; Yu, X.; Tian, J.; Wu, W. Preparation of Nitrogen-Doped Carbon Materials Based on Polyaniline Fiber and Their Oxygen Reduction Properties. Colloids and Surfaces A: Physicochemical and Engineering Aspects 2018, 539, 163-170. https://doi.org/10.1016/j.colsurfa.2017.12.024.

(15) Thanh, T. D.; Chuong, N. D.; Hien, H. V.; Kim, N. H.; Lee, J. H. CuAg@Ag Core-Shell Nanostructure Encapsulated by N-Doped Graphene as a High-Performance Catalyst for Oxygen Reduction Reaction. ACS Appl. Mater. Interfaces 2018, 10 (5), 4672-4681. https://doi.org/10.1021/acsami.7b16294.

(16) Sun, X.; Song, P.; Zhang, Y.; Liu, C.; Xu, W.; Xing, W. A Class of High Performance Metal-Free Oxygen Reduction Electrocatalysts Based on Cheap Carbon Blacks. Scientific Reports 2013, 3, 2505. https://doi.org/10.1038/srep02505.

(17) Han, H.; Chao, S.; Bai, Z.; Wang, X.; Yang, X.; Qiao, J.; Chen, Z.; Yang, L. MetalOrganic-Framework-Derived Co Nanoparticles Deposited on N-Doped Bimodal Mesoporous Carbon Nanorods as Efficient Bifunctional Catalysts for Rechargeable Zinc-Air Batteries. ChemElectroChem 2018, 5 (14), 1868-1873. https://doi.org/10.1002/celc.201701289.

(18) Yang, F.; Abadia, M.; Chen, C.; Wang, W.; Li, L.; Zhang, L.; Rogero, C.; Chuvilin, A.; Knez, M. Design of Active and Stable Oxygen Reduction Reaction Catalysts by Embedding CoxOynanoparticles into Nitrogen-Doped Carbon. Nano Res. 2017, 10 (1), 97-107. https://doi.org/10.1007/s12274-016-1269-5.

(19) Chen, R.; Yan, J.; Liu, Y.; Li, J. Three-Dimensional Nitrogen-Doped Graphene/MnO Nanoparticle Hybrids as a High-Performance Catalyst for Oxygen Reduction Reaction. $J$. Phys. Chem. C 2015, 119 (15), 8032-8037. https://doi.org/10.1021/acs.jpcc.5b00306.

(20) Li, X.; Guan, B. Y.; Gao, S.; Lou, X. W. (David). A General Dual-Templating Approach to Biomass-Derived Hierarchically Porous Heteroatom-Doped Carbon Materials for Enhanced Electrocatalytic Oxygen Reduction. Energy Environ. Sci. 2019, 12 (2), 648655. https://doi.org/10.1039/C8EE02779J.

(21) Xue, L.; Li, Y.; Liu, X.; Liu, Q.; Shang, J.; Duan, H.; Dai, L.; Shui, J. Zigzag Carbon as Efficient and Stable Oxygen Reduction Electrocatalyst for Proton Exchange Membrane Fuel Cells. Nature Communications 2018, 9 (1), 3819. https://doi.org/10.1038/s41467018-06279-x.

(22) Zhang, P.; Sun, F.; Xiang, Z.; Shen, Z.; Yun, J.; Cao, D. ZIF-Derived in Situ NitrogenDoped Porous Carbons as Efficient Metal-Free Electrocatalysts for Oxygen Reduction Reaction. Energy Environ. Sci. 2013, 7 (1), 442-450. https://doi.org/10.1039/C3EE42799D.

(23) Sathiskumar, C.; Ramakrishnan, S.; Vinothkannan, M.; Karthikeyan, S.; Yoo, D. J.; Rhan Kim, A. Nitrogen-Doped Porous Carbon Derived from Biomass Used as Trifunctional Electrocatalyst toward Oxygen Reduction, Oxygen Evolution and Hydrogen Evolution Reactions. Nanomaterials (Basel) 2019, 10 (1). https://doi.org/10.3390/nano10010076.

(24) Gao, R.; Dai, Q.; Du, F.; Yan, D.; Dai, L. C60-Adsorbed Single-Walled Carbon Nanotubes as Metal-Free, PH-Universal, and Multifunctional Catalysts for Oxygen Reduction, Oxygen Evolution, and Hydrogen Evolution. J. Am. Chem. Soc. 2019, 141 (29), 11658-11666. https://doi.org/10.1021/jacs.9b05006. 
(25) Han, L.; Cui, X.; Liu, Y.; Han, G.; Wu, X.; Xu, C. (Charles); Li, B. Nitrogen and Phosphorus Modification to Enhance the Catalytic Activity of Biomass-Derived Carbon toward the Oxygen Reduction Reaction. Sustainable Energy Fuels 2020, 4 (6), 27072717. https://doi.org/10.1039/C9SE00985J.

(26) Yang, H. B.; Miao, J.; Hung, S.-F.; Chen, J.; Tao, H. B.; Wang, X.; Zhang, L.; Chen, R.; Gao, J.; Chen, H. M.; Dai, L.; Liu, B. Identification of Catalytic Sites for Oxygen Reduction and Oxygen Evolution in N-Doped Graphene Materials: Development of Highly Efficient Metal-Free Bifunctional Electrocatalyst. Sci Adv 2016, 2 (4), e1501122. https://doi.org/10.1126/sciadv.1501122.

(27) Dumont, J. H.; Martinez, U.; Artyushkova, K.; Purdy, G. M.; Dattelbaum, A. M.; Zelenay, P.; Mohite, A.; Atanassov, P.; Gupta, G. Nitrogen-Doped Graphene Oxide Electrocatalysts for the Oxygen Reduction Reaction. ACS Appl. Nano Mater. 2019, 2 (3), 1675-1682. https://doi.org/10.1021/acsanm.8b02235.

(28) Liang, H.-W.; Wu, Z.-Y.; Chen, L.-F.; Li, C.; Yu, S.-H. Bacterial Cellulose Derived Nitrogen-Doped Carbon Nanofiber Aerogel: An Efficient Metal-Free Oxygen Reduction Electrocatalyst for Zinc-Air Battery. Nano Energy 2015, 11, 366-376. https://doi.org/10.1016/j.nanoen.2014.11.008.

(29) Gao, J.; Wang, Y.; Wu, H.; Liu, X.; Wang, L.; Yu, Q.; Li, A.; Wang, H.; Song, C.; Gao, Z.; Peng, M.; Zhang, M.; Ma, N.; Wang, J.; Zhou, W.; Wang, G.; Yin, Z.; Ma, D. Construction of a Sp3/Sp2 Carbon Interface in 3D N-Doped Nanocarbons for the Oxygen Reduction Reaction. Angewandte Chemie International Edition 2019, 58 (42), 1508915097. https://doi.org/10.1002/anie.201907915.

(30) Liu, M.; Song, Y.; He, S.; Tjiu, W. W.; Pan, J.; Xia, Y.-Y.; Liu, T. Nitrogen-Doped Graphene Nanoribbons as Efficient Metal-Free Electrocatalysts for Oxygen Reduction. ACS Appl. Mater. Interfaces 2014, 6 (6), 4214-4222. https://doi.org/10.1021/am405900r.

(31) Li, J.; Chen, S.; Yang, N.; Deng, M.; Ibraheem, S.; Deng, J.; Li, J.; Li, L.; Wei, Z. Ultrahigh-Loading Zinc Single-Atom Catalyst for Highly Efficient Oxygen Reduction in Both Acidic and Alkaline Media. Angewandte Chemie International Edition 2019, 58 (21), 7035-7039. https://doi.org/10.1002/anie.201902109.

(32) Yang, Q.; Jia, Y.; Wei, F.; Zhuang, L.; Yang, D.; Liu, J.; Wang, X.; Lin, S.; Yuan, P.; Yao, X. Understanding the Activity of Co-N4-xCx in Atomic Metal Catalysts for Oxygen Reduction Catalysis. Angewandte Chemie 2020, 132 (15), 6178-6183. https://doi.org/10.1002/ange.202000324.

(33) Yang, S.; Yu, Y.; Dou, M.; Zhang, Z.; Dai, L.; Wang, F. Two-Dimensional Conjugated Aromatic Networks as High-Site-Density and Single-Atom Electrocatalysts for the Oxygen Reduction Reaction. Angewandte Chemie International Edition 2019, 58 (41), 14724-14730. https://doi.org/10.1002/anie.201908023.

(34) Cui, L.; Cui, L.; Li, Z.; Zhang, J.; Wang, H.; Lu, S.; Xiang, Y. A Copper Single-Atom Catalyst towards Efficient and Durable Oxygen Reduction for Fuel Cells. J. Mater. Chem. A 2019, 7 (28), 16690-16695. https://doi.org/10.1039/C9TA03518D.

(35) Guo, C.; Li, Y.; Liao, W.; Liu, Y.; Li, Z.; Sun, L.; Chen, C.; Zhang, J.; Si, Y.; Li, L. Boosting the Oxygen Reduction Activity of a Three-Dimensional Network Co-N-C Electrocatalyst via Space-Confined Control of Nitrogen-Doping Efficiency and the Molecular-Level Coordination Effect. J. Mater. Chem. A 2018, 6 (27), 13050-13061. https://doi.org/10.1039/C8TA03759K. 
(36) Deng, Y.; Yin, S.; Liu, Y.; Lu, Y.; Cao, X.; Wang, L.; Wang, H.; Zhao, Y.; Gu, H. Mesoporous AgPdPt Nanotubes as Electrocatalysts for the Oxygen Reduction Reaction. ACS Appl. Nano Mater. 2019, 2 (4), 1876-1882. https://doi.org/10.1021/acsanm.8b02206.

(37) Xin, X.; Qin, H.; Cong, H.-P.; Yu, S.-H. Templating Synthesis of Mesoporous Fe3CEncapsulated Fe-N-Doped Carbon Hollow Nanospindles for Electrocatalysis. Langmuir 2018, 34 (17), 4952-4961. https://doi.org/10.1021/acs.langmuir.8b00548. 